\title{
Changes in hematologic indices in caucasian and non-caucasian pregnant women in the United States
}

\author{
Sarah K. Harm ${ }^{1}$, Mark H. Yazer ${ }^{1,2}$, Jonathan H. Waters ${ }^{3,4}$ \\ ${ }^{1}$ Department of Pathology, University of Pittsburgh Medical Center, ${ }^{2}$ The Institute for Transfusion Medicine, ${ }^{3}$ Department of \\ Anesthesiology, Magee Womens Hospital of the University of Pittsburgh Medical Center, ${ }^{4}$ Department of Bioengineering, University \\ of Pittsburgh, PA, USA
}

p-ISSN 1738-7949 / e-ISSN 2092-9129 http://dx.doi.org/10.5045/kjh.2012.47.2.136 Korean J Hematol 2012;47:136-41.

Received on April 12, 2012 Revised on May 2, 2012 Accepted on May 16, 2012

\section{Background}

The objective of this study was to determine if there are differences in common red blood cell (RBC) indices and platelet concentrations during pregnancy and to establish if any observed differences in these parameters were based on the patient's ethnicity.

\section{Methods}

From an electronic perinatal database which stores laboratory and clinical information on a large number of births at a regional hospital specializing in obstetrical care, RBC index and platelet concentration data were retrospectively analyzed at various time points throughout pregnancy. RBC index data was collected from 8,277 pregnant women (5,802 Caucasian pregnant women and 2,475 non-Caucasian pregnant women). Platelet concentration data was available from 8252 pregnant women $(5,784$ Caucasian pregnant women and 2,468 non-Caucasian pregnant women).

Results

Hemoglobin (HGB) levels were significantly higher amongst Caucasian women compared to non-Caucasian women $(P$ at least $<0.01)$ starting at 27 weeks gestation and proceeding until term. There was no significant difference in the mean PLT counts between Caucasian and non-Caucasian pregnant women at any point during gestation.

\section{Conclusion}

There are ethnic differences in HGB levels, but not the platelet concentrations, during pregnancy. Based on this finding it would be reasonable to conduct formal prospective studies to determine the clinical significance of this difference and to establish the threshold for diagnosing gestational anemia, especially in pregnant non-Caucasian women.

Key Words Anemia, Complete blood count, Hemoglobin, Pregnancy, Reference Ranges

\section{INTRODUCTION}

Physiologic changes in hemoglobin concentration (HGB) and platelet (PLT) count during pregnancy are well-known phenomena. [1, 2] The expansion of the pregnant woman's plasma volume as the pregnancy progresses compounded with a modest increase in the number of red blood cells and platelets results in a decrease in the HGB and PLT count during gestation relative to pre- and early pregnancy values.

The current Centers for Disease Control \& Prevention (CDC) reference ranges for HGB concentration and hema- tocrit (HCT) for pregnant women are based on older studies, including some from the 1970s, of a small number of European women who were receiving iron supplementation during their pregnancy [3-7]. Thus it is unclear whether the thresholds for anemia that were derived from these studies are applicable to modern day pregnant women in the USA. Currently the CDC threshold for diagnosing anemia during the first trimester is $11.0 \mathrm{~g} / \mathrm{dL}$, the second trimester is $10.5 \mathrm{~g} / \mathrm{dL}$, and $11.0 \mathrm{~g} / \mathrm{dL}$ during the third trimester [3]. In this report, the Magee Obstetrical Maternal and Infant (MOMI) database was used to determine if the criteria for gestational anemia and thrombocytopenia should be reconsidered in light of the small size of previous studies and

This is an Open Access article distributed under the terms of the Creative Commons Attribution Non-Commercial License (http://creativecommons.org/licenses/by-nc/3.0) which permits unrestricted non-commercial use, distribution, and reproduction in any medium, provided the original work is properly cited. 
to establish if there are different gestational HGB and PLT values based on ethnicity.

\section{MATERIALS AND METHODS}

At the Magee Womens Hospital (MWH), Pittsburgh, Pennsylvania, over 10,000 births occurred in 2010. At MWH, the Magee Obstetric Medical and Infant Database (MOMI) is available for research use. The database contains information on 115,000 births. Information within the database is coded from the labor and delivery medical record. Ethnicity was documented as either Caucasian or non-Caucasian. For the purpose of this study, information regarding gestational age was matched with records from the hospital laboratory information system. Access to the repository for this project was approved by the University of Pittsburgh's institutional review board.

For this analysis, all women who had antenatal complete blood count (CBC) parameters drawn between the years 2002 to 2008 were analyzed. During the study period, 48,470 live births occurred, and there were 9,766 unique women who had antenatal CBCs performed at MWH. Women who required antenatal laboratory testing were thus a subset of all of the women who delivered at this hospital. It is possible that some of the women who did not have blood tests performed at MWH had blood tests performed elsewhere. Since our goal was to analyze the $\mathrm{CBC}$ changes in a relatively healthy group of pregnant women, the cohort of women was normalized to those whose antenatal blood work was performed at MWH to those who did not have blood work performed at Magee based on the percentage of preterm deliveries. Preterm deliveries are a surrogate marker for high risk pregnancies. We then randomly selected the percentage of preterm deliveries amongst the women who had blood drawn at MWH during their pregnancy and verified that it was equal to the percentage of women with preterm delivery who did not have antenatal blood work drawn at MWH. RBC data was collected from 8,277 pregnant women $(5,802$ Caucasian pregnant women and 2,475 non-Caucasian pregnant women). PLT data was available from 8252 pregnant women (5,784 Caucasian pregnant women and 2,468 non-Caucasian pregnant women).

CBC information was collected and stratified by both gestational age and ethnicity. The differences between these parameters were evaluated using a two-way ANOVA (Graphpad software). A $P$ value $<0.05$ was considered statistically significant.

\section{RESULTS}

\section{Hemoglobin (HGB) and erythrocyte indices}

The mean HGB values declined in the first and second trimesters and reached a nadir of $11.6 \mathrm{~g} / \mathrm{dL}( \pm 1.2 \mathrm{~g} / \mathrm{dL})$ (Fig. $1 \mathrm{~A})$ at 24 weeks gestation in the Caucasian population. In these women the mean HGB values declined in the first through second trimesters and slowly rose during the third trimester. Unlike in the Caucasian population, a clear HGB nadir during the second trimester was not appreciated in the non-Caucasian population. The mean HGB values of the non-Caucasian pregnant women were significantly lower than the mean values of the Caucasian pregnant women starting at 27 weeks gestation and continuing through term ( $P$ at least $<0.01$ at all time points). The HCT values followed a similar trend; the mean HCT values in the non-Caucasian pregnant women were significantly lower than the mean values in Caucasian pregnant women starting at week 30 and continuing through week $39(P$ at least $<0.05$ at all time points), with the exception of week 36 where the HCT values were not significantly different.

The red blood cell (RBC) count, red cell distribution width (RDW), mean corpuscular volume (MCV), mean corpuscular hemoglobin $(\mathrm{MCH})$, and mean corpuscular hemoglobin concentration (MCHC) are plotted in Fig. 2. There was no significant differences in the mean RBC counts between the Caucasian and non-Caucasian pregnant women throughout the gestational period ( $P>0.05$ at all time points). The mean RDW values in non-Caucasian women were significantly higher than the mean values of Caucasian pregnant women at gestational weeks 7, 13, 31, and between weeks 35-38 $(P$ at least $<0.05)$. The mean $\mathrm{MCV}$ and $\mathrm{MCH}$ values in Caucasian pregnant women were significantly higher than the means of non-Caucasian pregnant women between weeks 30-39 ( $P$ at least $<0.01$ for both parameters at all time points), while the MCHC was significantly higher amongst the Caucasian women starting at week 18 and continuing through week $39(P$ at least $<0.001)$.

\section{Platelets (PLT)}

The mean PLT count gradually decreased throughout the gestational period in both Caucasian and non-Caucasian women (Fig. 1C). The mean PLT count reached a nadir of $229 \times 10^{9} / \mathrm{L}\left( \pm 61 \times 10^{9} / \mathrm{L}\right)$ at 38 weeks gestation in Caucasian pregnant women. In non-Caucasian pregnant women, the nadir of $224 \times 10^{9} / \mathrm{L}\left( \pm 71 \times 10^{9} / \mathrm{L}\right)$ occurred at 37 weeks gestation. There was no significant difference in the mean PLT counts between Caucasian and non-Caucasian pregnant women throughout the gestational period $(P>0.05$ at all time points).

\section{DISCUSSION}

According to the CDC, threshold for diagnosing anemia during the first trimester is $11.0 \mathrm{~g} / \mathrm{dL}$, the second trimester is $10.5 \mathrm{~g} / \mathrm{dL}$, and $11.0 \mathrm{~g} / \mathrm{dL}$ during the third trimester [3]. These thresholds are based on a small number of studies of European women who had their HGB measured at only a few time points during gestation, and do not account for any potential ethnic differences amongst pregnant women [3-7] (Table 1). In spite of these limitations, the data presented here suggests that the current anemia thresholds are reasonable for diagnosing anemia in Caucasian pregnant women. 


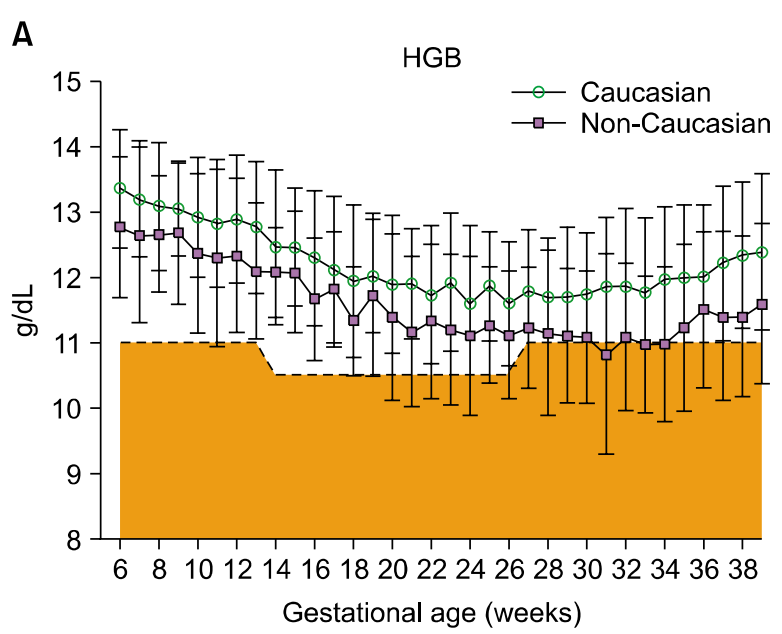

C

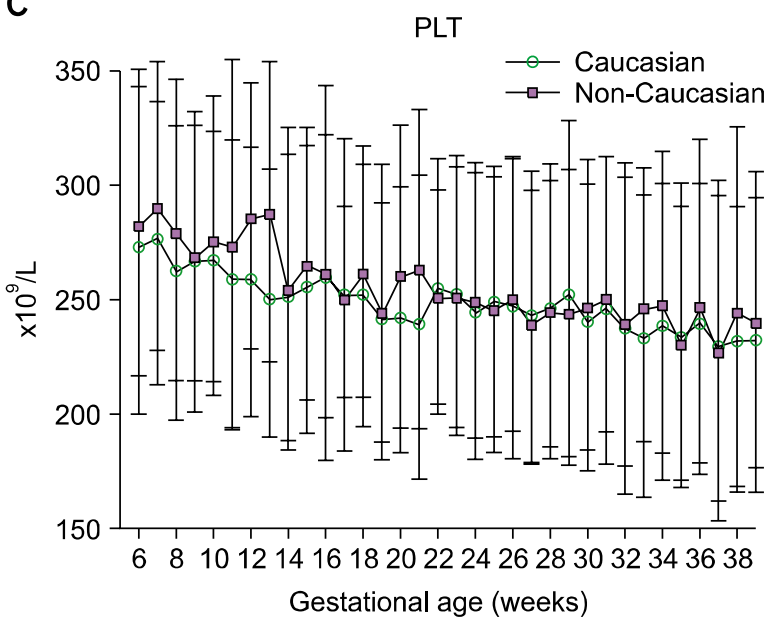

B

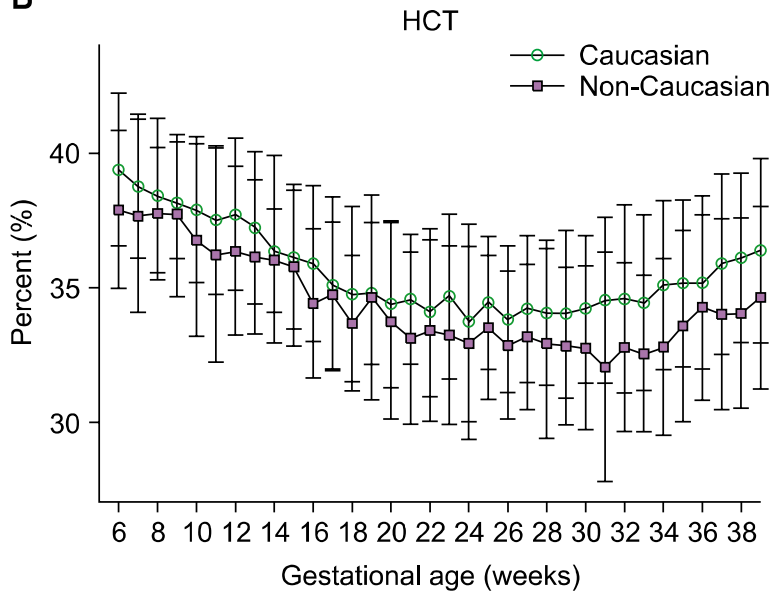

D

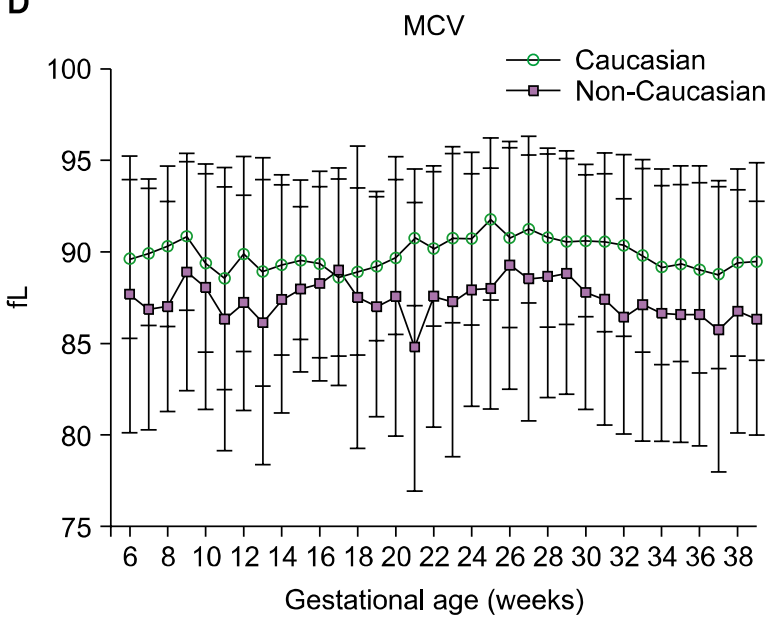

Fig. 1. Hemoglobin (HGB), hematocrit (HCT), platelet count (PLT), and mean corpuscular volume (MCV) in Caucasian ( $($ ) and non-Caucasian ( $\square$ ) pregnant women throughout the gestational period. Mean \pm 1 SD are shown. See text for statistical analysis. The shaded area on the HGB graph represents the $\mathrm{CDC}^{\prime}$ s recommended threshold for anemia during pregnancy.

However, based on this study, many non-Caucasian pregnant women would be diagnosed with gestational anemia if the current CDC thresholds were applied, especially in the third trimester. Thus there are ethnic differences between Caucasians and non-Caucasians in HGB during pregnancy, an observation of some recent studies in different ethnic populations [8-10] (Table 1). Additionally, while an obvious HGB nadir was not readily apparent amongst the nonCaucasian women (it is likely that the value at 31 weeks is spuriously low as the HGB value drops precipitously after week 30 and rose again by week 32), the HGB levels amongst non-Caucasian women appeared to recover more slowly as the third trimester progressed relative to the rate of recovery in the Caucasian population. It is also interesting to note that the smooth trajectory of the changes in the HGB values for the Caucasian women was not seen in the context of the non-Caucasian women. The explanation for these observations is unknown and merits further study, although part of the variability in the course of the decline and sub- sequent rise in HGB in the non-Caucasian women could have been due to the mix of ethnicities that were subsumed in the term "non-Caucasian" - if ethnic differences amongst these individuals exist.

Regardless of the mean HGB level, both the Caucasian and non-Caucasian women demonstrated some variability in the HGB levels at all gestational time points. Furthermore there were significant differences not only in the HGB but also in the HCT, RDW, MCV, MCH, and MCHC values between Caucasians and non-Caucasians in our study, with Caucasian pregnant women tending to have higher mean values than non-Caucasian pregnant women in all parameters except the RDW. A previous study demonstrated that the ranges of HGB and HCT were lower among non-pregnant black women than in non-pregnant Caucasian women even after adjustment for income [11]. In addition, these differences are not necessarily caused by a difference in iron status $[11,12]$. Thus the differences in the HGB and other erythrocyte parameters may be reflective of true ethnic and pop- 


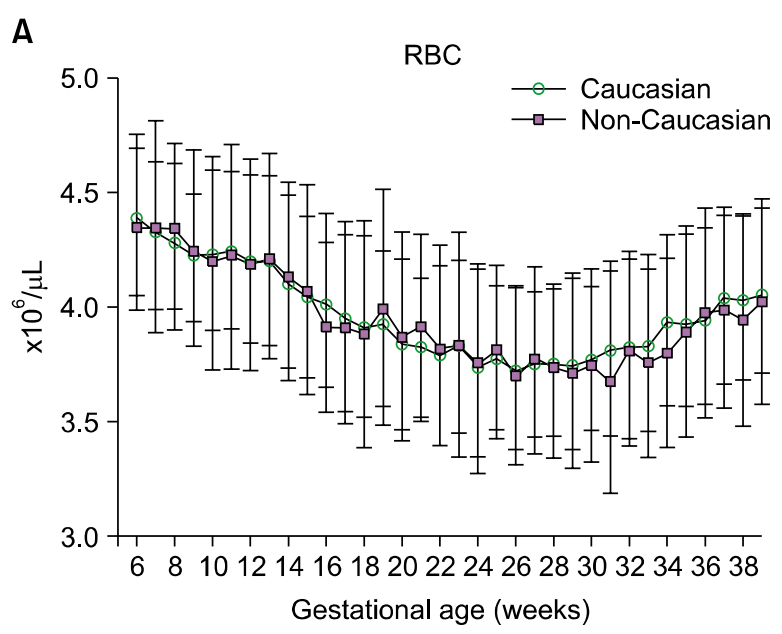

C

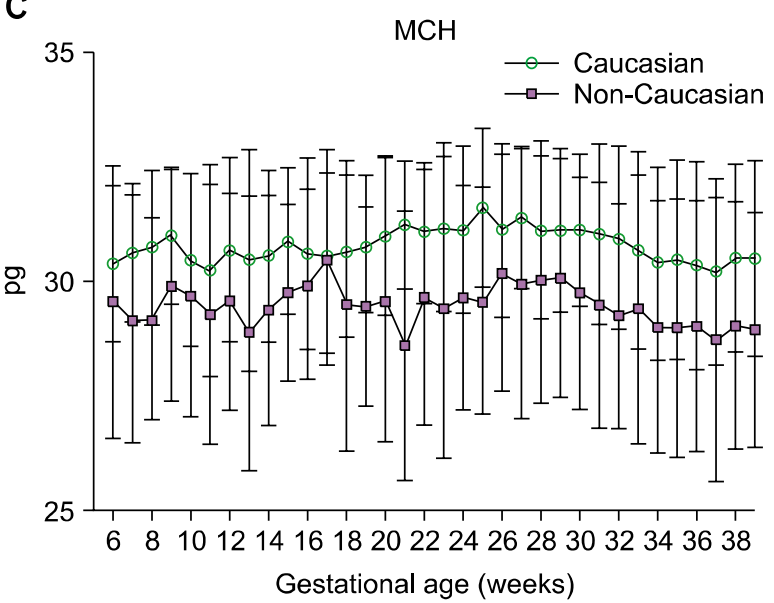

B

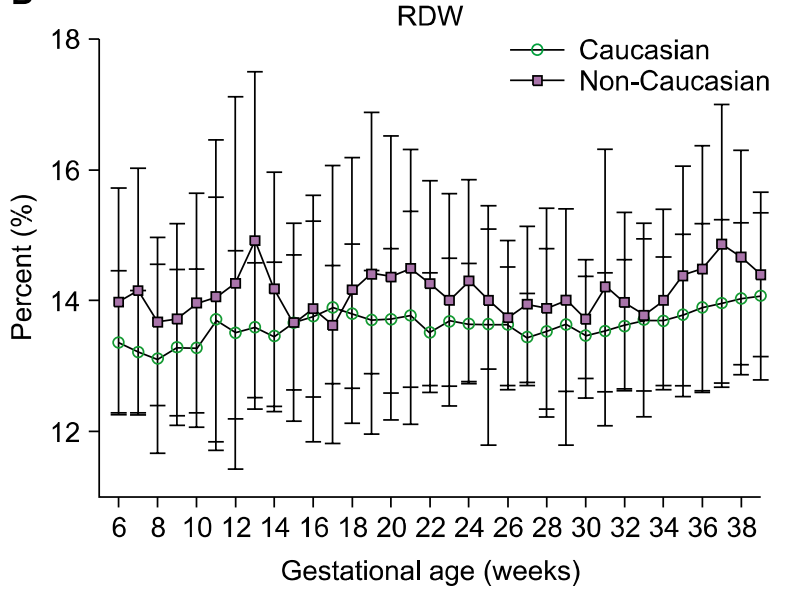

D

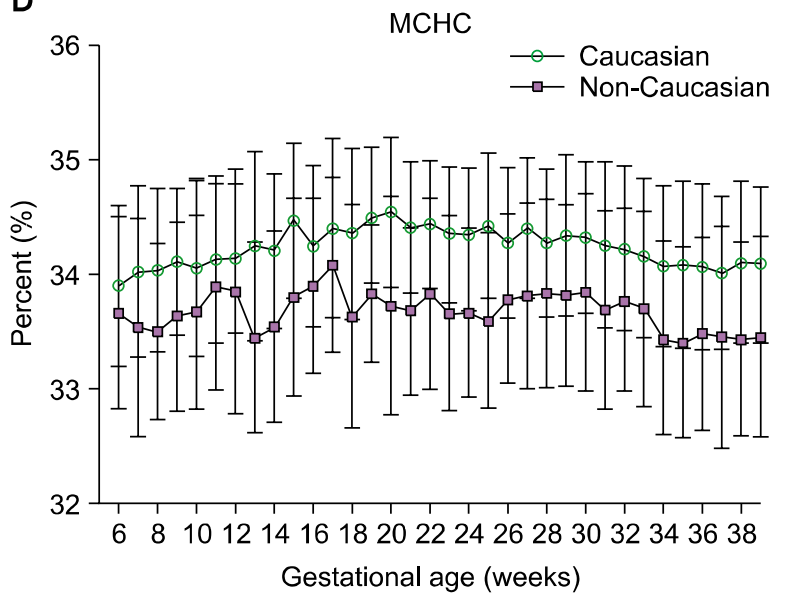

Fig. 2. Red blood cell count (RBC), red cell distribution width (RDW), mean corpuscular hemoglobin (MCH), and mean corpuscular hemoglobin concentration $(\mathrm{MCHC})$, in Caucasian (०) and non-Caucasian ( $\square$ ) pregnant women throughout the gestational period. Mean \pm 1 SD are shown. See text for statistical analysis.

ulation differences that are not well understood at this time. Although the clinical significance of these differences is not known, that they exist suggests that different thresholds could be used for diagnosing anemia in Caucasian and non-Caucasian pregnant patients.

The gradual decrease in the PLT count throughout gestation is reflective of the increased plasma volume during a healthy pregnancy and increased utero-placental consumption during the third trimester [13]. In a stable, non-bleeding pregnant woman without a bleeding diathesis, the American College of Obstetricians and Gynecologists (ACOG) currently defines mild maternal thrombocytopenia as a platelet count between $70 \times 10^{9} / \mathrm{L}$ to $150 \times 10^{9} / \mathrm{L}[14]$. In our generally healthy pregnant patient population, the mean $\mathrm{PLT}$ value reached a nadir of $229 \times 10^{9} / \mathrm{L}\left( \pm 61 \times 10^{9} / \mathrm{L}\right)$ at 38 weeks gestation in Caucasian pregnant women and a nadir of $224 \times 10^{9} / \mathrm{L}\left( \pm 71 \times 10^{9} / \mathrm{L}\right)$ at 37 weeks gestation in nonCaucasian pregnant women. The lowest PLT counts reported here (mean -1 SD) were $168 \times 10^{9} / \mathrm{L}$ in Caucasian pregnant women and $153 \times 10^{9} / \mathrm{L}$ in non-Caucasian pregnant women, both at 37 weeks, demonstrating that a small number of pregnant women, independent of race, are close to having mild maternal thrombocytopenia at parturition.

A recent study showed that approximately $8 \%$ of healthy pregnant women had PLT counts between $97 \times 10^{9} / \mathrm{L}-$ $150 \times 10^{9} / \mathrm{L}$ at delivery, consistent with the ACOG definition of mild maternal thrombocytopenia [15]. A majority of those healthy pregnant women had a PLT count between $100 \times 10^{9} / \mathrm{L}-150 \times 10^{9} / \mathrm{L}$ at delivery, with approximately $5 \%$ having platelet counts $<100 \times 10^{9} / \mathrm{L}$, and all exhibited no signs of bleeding or platelet dysfunction [16]. Furthermore, the same study suggested that healthy women at delivery who have mild maternal thrombocytopenia during their pregnancy require no specific treatment, and decisions like the mode of delivery and the placement of epidural analgesia, should be determined by clinical indications only. Other studies support these findings [17-19]. Thus recognizing that normal healthy pregnant women may have platelet counts of approximately $150 \times 10^{9} / \mathrm{L}$ without harboring an increased risk of bleeding to themselves or their fetus can help guide 


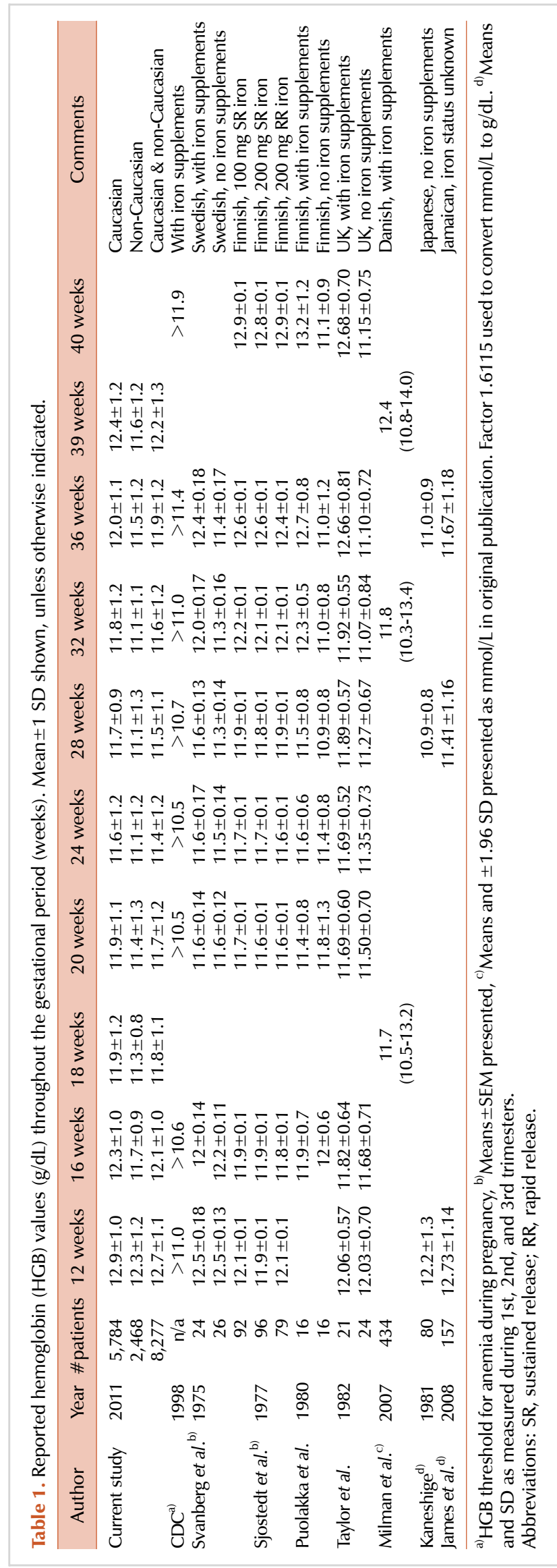

both the decision to insert an epidural, and their delivery management. A prospective study to formally test this hypothesis is required.

As this was a retrospective review of a large maternal database, one limitation of this study is that it is unknown if the women were taking any vitamin and/or mineral supplementation during their pregnancy which might confound the measurement of HGB during gestation by inflating the value. Iron supplementation is currently recommended during pregnancy thus it is reasonable to conclude that many of the women in this study were taking this supplement. However, studies have shown that iron supplementation may only be effective in reversing iron-deficiency anemia after the $25^{\text {th }}$ week of gestation and may only increase the HGB by $1 \mathrm{~g} / \mathrm{dL}$ at term [5, 7, 20]. Iron supplementation is currently (and should continue to be) recommended during pregnancy. Also, given the large sample size, if there were small groups of women with different physiologies, then their unique characteristics might not be reflected in the group's mean values. Thus the ranges demonstrated in Fig. 1 and 2 might not apply to women who have known elemental absorption problems with their gastroenterological tracts, or disease of the bone marrow that prevent them from producing RBCs. Furthermore, the exact distribution of women who were healthy throughout their pregnancies and those who experienced maternal or fetal complications in this study is not known, however the sample cohort was intentionally selected to be representative of the general maternal population at our hospital. Lastly, the exact distribution of ethnicities subsumed in the term "nonCaucasian" in this study is not known because of the non-specific manner in which this data is collected. However, based on data from the 2010 census data, $82 \%$ of the population of Allegheny county in which Pittsburgh is located were Caucasian, $13 \%$ were black, $<3 \%$ were Asian, while $<4 \%$ in total self identified as either American Indian, Hispanic or of mixed race [21]. Thus the majority of those reported as non-Caucasian were likely black.

We have demonstrated that there are important ethnic differences in various CBC parameters between Caucasian and non-Caucasian pregnant women during pregnancy. Potentially the most important difference is that of the HGB values in the third trimester which suggest that the currently employed threshold for diagnosing anemia in non-Caucasian pregnant women should be reconsidered. Formal prospective studies to determine the clinical impact of the differences in these parameters is required.

\section{ACKNOWLEDGEMENTS}

The authors thank Marijane Krohn, PhD from the Department of Obstetrics and Gynecology, University of Pittsburgh, Pennsylvania for statistical analysis of the data. 


\section{REFERENCES}

1. Sifakis S, Pharmakides G. Anemia in pregnancy. Ann N Y Acad Sci 2000;900:125-36.

2. Lund CJ, Donovan JC. Blood volume during pregnancy. Significance of plasma and red cell volumes. Am J Obstet Gynecol 1967;98:394-403.

3. Recommendations to prevent and control iron deficiency in the United States. Centers for Disease Control and Prevention. MMWR Recomm Rep 1998;47:1-29.

4. Svanberg B, Arvidsson B, Norrby A, Rybo G, Sölvell L. Absorption of supplemental iron during pregnancy - a longitudinal study with repeated bone-marrow studies and absorption measurements. Acta Obstet Gynecol Scand Suppl 1975;48:87-108.

5. Sjostedt JE, Manner P, Nummi S, Ekenved G. Oral iron prophylaxis during pregnancy: a comparative study on different dosage regimens. Acta Obstet Gynecol Scand Suppl 1977;60:3-9.

6. Taylor DJ, Mallen C, McDougall N, Lind T. Effect of iron supplementation on serum ferritin levels during and after pregnancy. Br J Obstet Gynaecol 1982;89:1011-7.

7. Puolakka J, Jänne O, Pakarinen A, Järvinen PA, Vihko R. Serum ferritin as a measure of iron stores during and after normal pregnancy with and without iron supplements. Acta Obstet Gynecol Scand Suppl 1980;95:43-51.

8. Milman N, Bergholt T, Byg KE, Eriksen L, Hvas AM. Reference intervals for haematological variables during normal pregnancy and postpartum in 434 healthy Danish women. Eur J Haematol 2007;79:39-46.

9. Kaneshige E. Serum ferritin as an assessment of iron stores and other hematologic parameters during pregnancy. Obstet Gynecol 1981;57:238-42.

10. James TR, Reid HL, Mullings AM. Are published standards for haematological indices in pregnancy applicable across populations: an evaluation in healthy pregnant Jamaican women. BMC Pregnancy Childbirth 2008;8:8.
11. Johnson-Spear MA, Yip R. Hemoglobin difference between black and white women with comparable iron status: justification for race-specific anemia criteria. Am J Clin Nutr 1994;60:117-21.

12. Perry GS, Byers T, Yip R, Margen S. Iron nutrition does not account for the hemoglobin differences between blacks and whites. J Nutr 1992;122:1417-24.

13. Hellgren M. Hemostasis during normal pregnancy and puerperium. Semin Thromb Hemost 2003;29:125-30.

14. ACOG practice bulletin: Thrombocytopenia in pregnancy. Number 6, September 1999. Clinical management guidelines for obstetrician-gynecologists. American College of Obstetricians and Gynecologists. Int J Gynaecol Obstet 1999;67:117-28.

15. Burrows RF, Kelton JG. Incidentally detected thrombocytopenia in healthy mothers and their infants. N Engl J Med 1988;319: 142-5.

16. Burrows RF, Kelton JG. Thrombocytopenia at delivery: a prospective survey of 6715 deliveries. Am J Obstet Gynecol 1990;162:731-4.

17. Samuels P, Bussel JB, Braitman LE, et al. Estimation of the risk of thrombocytopenia in the offspring of pregnant women with presumed immune thrombocytopenic purpura. N Engl J Med 1990;323:229-35.

18. Aster RH. "Gestational" thrombocytopenia: a plea for conservative management. N Engl J Med 1990;323:264-6.

19. Hart D, Dunetz C, Nardi M, Porges RF, Weiss A, Karpatkin M. An epidemic of maternal thrombocytopenia associated with elevated antiplatelet antibody. Platelet count and antiplatelet antibody in 116 consecutive pregnancies: relationship to neonatal platelet count. Am J Obstet Gynecol 1986;154:878-83.

20. Romslo I, Haram K, Sagen N, Augensen K. Iron requirement in normal pregnancy as assessed by serum ferritin, serum transferrin saturation and erythrocyte protoporphyrin determinations. Br J Obstet Gynaecol 1983;90:101-7.

21. US Census Bureau. State \& County Quickfacts. Suitland, MD: US Census Bureau. (Accessed December 6, 2011, at http://quickfacts. census.gov/qfd/states/42/42003.html.) 